\title{
Super-resolution Time Delay Estimation in Multipath Environments ${ }^{\dagger}$
}

\author{
Feng-Xiang $\mathrm{Ge}^{2}$, Dongxu Shen ${ }^{2}$, Yingning Peng ${ }^{1}$, and Victor O. K. $\mathrm{Li}^{2}$ \\ 1. Dept. of Electronic Engineering, Tsinghua University, Beijing, 100084, China, \\ 2. Dept. of Electrical \& Electronic Engineering, The University of Hong Kong, Hong Kong, China
}

\begin{abstract}
The problem of super-resolution time delay estimation in multipath environments is addressed in this paper. Two cases, active and passive systems, are considered. The time delay estimation is first converted into a sinusoidal parameter estimation problem. Then the sinusoidal parameters are estimated by generalizing the Multiple Signal Classification (MUSIC) algorithm for single-experiment data. The proposed method, referred to as the MUSIC-type algorithm, approximates the Cramer-Rao bound (CRB) in terms of the mean square errors (MSEs) for different signal-to-noise ratios (SNRs) and separations of multipath components. Simulation results show that the MUSIC-type algorithm performs better than the classical correlation approach and the conventional MUSIC method for the closely spaced components in multipath environments.
\end{abstract}

Index Terms - time delay estimation, multipath environments, Eigenanalysis, Sequential Quadratic Programming

\section{INTRODUCTION}

The time delay estimation is a fundamental signal processing problem with application in many areas, such as radar [1], sonar [1], communications [2], etc.. The classical time delay estimation techniques are based on correlations [1], and are only effective when multipath components are well separated in arrival time or when only one component is present in the received signal. Their performance will degrade significantly when the signal components are closely spaced in multipath environments.

A number of super-resolution techniques that can separate closely-spaced multipath components have been developed. Initially, the problem was studied in the time domain [3]. However, they offer marginal performance gain against the classical methods. Hou and Wu [4] first proposed a modelbased sinusoidal estimation method, which converts the time delay estimation problem into a sinusoidal parameter estimation problem. Several other model-based sinusoidal estimation methods were also presented in [5], [6]. These methods involve a spectral-division operation, so they are only suitable for signals with flat spectra [7], [8]. The approaches in [9]-[11] can be applied to signals with non-flat spectra. However, these methods do not guarantee global convergence and require large numbers of data samples.

In this paper, a super-resolution time delay estimation method is proposed, based on a generalization of the Multiple

$\dagger$ This work was supported in part by the Innovation Fund (No. 091205056) of the School of Information Science and Technology at Tsinghua University and in part by the Research Grant Council of Hong Kong, under grant No. HKU 7047/00E.
Signal Classification (MUSIC) algorithm [17]. Two cases, active and passive systems, are considered. In particular, the transmitted signal is always unknown in passive systems. Our method is applicable to signals with non-flat spectra, as opposed to the model-based sinusoidal estimation methods [4][6]. In addition, our method requires much fewer samples than those in [9]-[11]. The performance of the proposed time delay estimator is evaluated by numerical simulations. The mean square errors (MSE) for different SNRs and the time delay separations in a multipath environment are shown to approximate the Cramer-Rao bound (CRB), and the proposed estimator performs better than the classical correlation approach and the conventional MUSIC method.

This paper is organized as follows. Section 2 presents the data models for estimating the time delay. In Section 3, we derive the super-resolution time delay estimators. Simulation results are shown in Section 4. Conclusions are provided in Section 5 .

\section{DATA MODEL}

In this section, we give the data models for the time delay estimation. First, the notations in this paper are listed as follows:

$$
\begin{aligned}
*, & \text { complex conjugate, } \\
T, & \text { transpose, } \\
H, & \text { Hermitian transpose, } \\
\operatorname{Re}(\cdot) \text { and } \operatorname{Im}(\cdot), & \text { real part and imaginary part }, \\
E(\cdot), & \text { expected value, } \\
\|\cdot\|, & \text { Euclidean norm, } \\
\mathrm{DFT}^{-1}, & \text { discrete Fourier transform (DFT), } \\
\mathrm{DFT}^{-1}, & \text { inverse DFT (IDFT). }
\end{aligned}
$$

\section{A. The Data Model in Active Systems}

In active systems, the time delay estimation is always accomplished by matched filter or cross-correlation where the reference signal is the known transmitted signal. Suppose that the received signal is described as

$$
r(t)=\sum_{i=1}^{D} \lambda_{i} s\left(t-\tilde{\tau}_{i}\right)+w(t), \quad 0 \leq t \leq T_{r},
$$

where $D$ is the number of multipath components, $s(t)$ is the transmitted signal with duration $T_{s}, \lambda_{i}$ and $\tilde{\tau}_{i}$ are the corresponding random amplitudes and time delays, $w(t)$ is 
additive white Gaussian noise (AGWN), $T_{r}$ is the duration of $r(t)$. The resulting discrete-time sequence can be written as

$$
r(n)=\sum_{i=1}^{D} \lambda_{i} s\left(n-\tau_{i}\right)+w(n), \quad n=0,1, \cdots, K_{r}-1,
$$

where $\tau_{i}, i=1,2, \cdots, D$, are the discrete time delays, and $K_{r}$ is the length of $r(n)$. We zero-pad $s(n)$ and $r(n)$ to length $K_{x_{A}}=K_{r}+K_{s}-1$, where $K_{s}$ is the length of $s(n)$. Then by computing the circular correlation between $s(n)$ and $r(n)$, and carrying out the square envelope detection, we have

$$
\begin{aligned}
\left|R_{A}(\tau)\right|^{2}= & \left|\sum_{n=0}^{K_{x_{A}}-1} s(n) \cdot r^{*}(n+\tau)\right|^{2} \\
= & \mid \sum_{k=0}^{K_{x_{A}}-1}\left\{\sum_{i=1}^{D} \lambda_{i}^{*}|S(k)|^{2} e^{j 2 \pi k \tau_{i} / K_{x_{A}}}\right. \\
& \left.\quad+S(k) \cdot W^{*}(k)\right\} e^{-j 2 \pi k \tau /\left.K_{x_{A}}\right|^{2}}
\end{aligned}
$$

where $S(k)$ and $W(k)$ are the discrete Fourier transforms of $s(n)$ and $w(n)$, respectively. We denote

$$
x_{A}(k)=\sum_{i=1}^{D} \lambda_{i}^{*}|S(k)|^{2} e^{j 2 \pi k \tau_{i} / K_{x_{A}}}+S(k) \cdot W^{*}(k),
$$

and have the following relation,

$$
\left|R_{A}(\tau)\right|^{2}=\left(\operatorname{DFT}\left[x_{A}(k)\right]\right)^{2} .
$$

In vector form, (4) is given by

$$
\begin{aligned}
\mathbf{x}_{A} & =\sum_{i=1}^{D} \lambda_{i}^{*} \Lambda\left(\tau_{i}\right) \mathbf{S}+\mathbf{W}_{A} \\
& =\mathbf{\Phi}_{A} \boldsymbol{\lambda}_{A}+\mathbf{W}_{A}
\end{aligned}
$$

where

$$
\begin{aligned}
& \mathbf{x}_{A}=\left[\begin{array}{llll}
x_{A}(0) & x_{A}(1) & \cdots & x_{A}\left(K_{x_{A}}-1\right)
\end{array}\right]^{T} \text {, } \\
& \boldsymbol{\lambda}_{A}=\left[\begin{array}{llll}
\lambda_{1}^{*} & \lambda_{2}^{*} & \cdots & \lambda_{D}^{*}
\end{array}\right]^{T} \text {, } \\
& \boldsymbol{\Lambda}\left(\tau_{i}\right)=\operatorname{diag}\left(\begin{array}{cccc}
1 & e^{\frac{j 2 \pi \tau_{i}}{K_{x}}} & \cdots & e^{\frac{j 2 \pi\left(K_{x_{A}}-1\right) \tau_{i}}{K_{x_{A}}}}
\end{array}\right) \text {, } \\
& \mathbf{S}=\left[\begin{array}{llll}
|S(0)|^{2} & |S(1)|^{2} & \cdots & \left|S\left(K_{x_{A}}-1\right)\right|^{2}
\end{array}\right]^{T}, \\
& \boldsymbol{\Phi}_{A}=\left[\begin{array}{llll}
\boldsymbol{\Lambda}\left(\tau_{1}\right) \mathbf{S} & \boldsymbol{\Lambda}\left(\tau_{2}\right) \mathbf{S} & \cdots & \boldsymbol{\Lambda}\left(\tau_{D}\right) \mathbf{S}
\end{array}\right], \\
& \mathbf{W}_{A}=\left[S(0) \cdot W^{*}(0) \quad S(1) \cdot W^{*}(1)\right. \\
& \text {.. } \left.\quad S\left(K_{x_{A}}-1\right) \cdot W^{*}\left(K_{x_{A}}-1\right)\right]^{T} \text {. }
\end{aligned}
$$

\section{B. The Data Model in Passive Systems}

In passive systems, signals are received at spatially separated sensors and their time difference of arrival are always measured by cross-correlation, where the reference signal is one of the received signals. Suppose that two received signals at two spatially separated sensors can be modeled as

$$
\left\{\begin{array}{l}
r_{1}(t)=\sum_{j=1}^{D_{1}} \lambda_{1 j} \cdot s\left(t-\tilde{\tau}_{1 j}\right)+w_{1}(t) \\
r_{2}(t)=\sum_{i=1}^{D_{2}} \lambda_{2 i} \cdot s\left(t-\tilde{\tau}_{2 i}\right)+w_{2}(t)
\end{array}, 0 \leq t \leq T_{r},\right.
$$

where $D_{1}$ and $D_{2}$ are the number of multipaths, $\tilde{\tau}_{1 j}$ and $\tilde{\tau}_{2 i}$ are the time delays of the received signals for each multipath, $\lambda_{1 j}$ and $\lambda_{2 i}$ are the random amplitudes which are mutually uncorrelated, $w_{1}(t)$ and $w_{2}(t)$ are AWGNs, respectively.
For the sake of simplicity, we assume $r_{1}(t)$ has one signal component, i.e., $D_{1}=1$, and $r_{2}(t)$ has multiple signal components, i.e., $D_{2}=D>1$, which are to be resolved by the proposed super-resolution algorithm. Nevertheless, our results can be easily extended to the cases of $D_{1}>1$ using the auxiliary processing in [4]. Then the resulting discrete-time sequences can be written as

$$
\left\{\begin{array}{r}
r_{1}(n)=\lambda_{11} \cdot s\left(n-\tau_{11}\right)+w_{1}(n) \\
r_{2}(n)=\sum_{i=1}^{D} \lambda_{2 i} \cdot s\left(n-\tau_{2 i}\right)+w_{2}(n) \\
n=0,1, \cdots, K_{r}-1
\end{array}\right.
$$

where $\tau_{11}$ and $\tau_{2 i}, i=1,2, \cdots, D$, are the discrete time delays, and $K_{r}$ is the length of the sequences.

The above two discrete-time sequences are then zero-padded to length $K_{x_{P}}=2 K_{r}-1$. Let $W_{1}(k)$ and $W_{2}(k)$ be the discrete Fourier transform of $w_{1}(t)$ and $w_{2}(t)$, respectively. By computing the circular correlation function between $r_{1}(t)$ and $r_{2}(t)$, and carrying out square envelope detection, we have

$$
\begin{aligned}
\left|R_{P}(\tau)\right|^{2}= & \left|\sum_{n=0}^{K_{x_{P}}-1} r_{1}(n) \cdot r_{2}^{*}(n+\tau)\right|^{2} \\
= & \mid \sum_{k=0}^{K_{x_{P}}-1}\left\{\sum_{i=1}^{D} \lambda_{11} \lambda_{2 i}^{*}|S(k)|^{2} e^{\frac{j 2 \pi k\left(\tau_{2 i}-\tau_{11}\right)}{K_{x_{P}}}}\right. \\
& +\lambda_{11} S(k) \cdot e^{\frac{-j 2 \pi k \tau_{11}}{K_{x_{P}}}} W_{2}^{*}(k)+W_{1}(k) \cdot W_{2}^{*}(k) \\
& \left.+W_{1}(k) \sum_{i=1}^{D} \lambda_{2 i}^{*} S^{*}(k) e^{\frac{j 2 \pi k \tau_{2 i}}{K_{x_{P}}}}\right\}\left.e^{\frac{-j 2 \pi k \tau}{K_{x_{P}}}}\right|^{2}
\end{aligned}
$$

We denote

$$
\begin{aligned}
x_{P}(k)= & \sum_{i=1}^{D} \lambda_{11} \lambda_{2 i}^{*}|S(k)|^{2} e^{\frac{j 2 \pi k \Delta \tau_{i}}{K_{x_{P}}}} \\
& +\lambda_{11} S(k) \cdot e^{\frac{-j 2 \pi k \tau_{11}}{K_{x_{P}}}} W_{2}^{*}(k)+W_{1}(k) \cdot W_{2}^{*}(k) \\
& +W_{1}(k) \sum_{i=1}^{D} \lambda_{2 i}^{*} S^{*}(k) e^{\frac{j 2 \pi k \tau_{2 i}}{K_{x_{P}}}} \\
= & \sum_{i=1}^{D} \lambda_{11} \lambda_{2 i}^{*}|S(k)|^{2} e^{\frac{j 2 \pi k \Delta \tau_{i}}{K_{x_{P}}}}+W_{P}(k),
\end{aligned}
$$

where $\Delta \tau_{i}=\tau_{2 i}-\tau_{11}, i=1, \cdots, D$, which are the time difference of arrivals to be estimated, and $W_{P}(k)=\lambda_{11} S(k)$. $e^{\frac{-j 2 \pi k \tau_{11}}{K_{x_{P}}}} W_{2}^{*}(k)+W_{1}(k) \sum_{i=1}^{D} \lambda_{2 i}^{*} S^{*}(k) e^{\frac{j 2 \pi k \tau_{2 i}}{K_{x_{P}}}}+W_{1}(k)$. $W_{2}^{*}(k)$. Then we have the following relation

$$
\left|R_{P}(\tau)\right|^{2}=\left(\operatorname{DFT}\left[x_{P}(k)\right]\right)^{2} .
$$

In vector form, (10) is given by

$$
\begin{aligned}
\mathbf{x}_{P} & =\sum_{i=1}^{D} \lambda_{11} \lambda_{2 i}^{*} \Lambda\left(\Delta \tau_{i}\right) \mathbf{S}+\mathbf{W}_{P} \\
& =\mathbf{\Phi}_{P} \boldsymbol{\lambda}_{P}+\mathbf{W}_{P}
\end{aligned}
$$

where

$$
\begin{aligned}
& \mathbf{x}_{P}=\left[\begin{array}{llll}
x_{P}(0) & x_{P}(1) & \cdots & x_{P}\left(K_{x_{P}}-1\right)
\end{array}\right]^{T}, \\
& \boldsymbol{\lambda}_{P}=\lambda_{11} \cdot\left[\begin{array}{llll}
\lambda_{21}^{*} & \lambda_{22}^{*} & \cdots & \lambda_{2 D}^{*}
\end{array}\right]^{T}, \\
& \boldsymbol{\Lambda}\left(\Delta \tau_{i}\right)=\operatorname{diag}\left(\begin{array}{cccc}
1 & e^{j 2 \pi \frac{\Delta \tau_{i}}{K_{P}}} & \cdots & e^{j 2 \pi \frac{\left(K_{x_{P}}-1\right) \Delta \tau_{i}}{K_{x_{P}}}}
\end{array}\right), \\
& \boldsymbol{\Phi}_{P}=\left[\begin{array}{lllll}
\boldsymbol{\Lambda}\left(\Delta \tau_{1}\right) \mathbf{S} & \boldsymbol{\Lambda}\left(\Delta \tau_{2}\right) \mathbf{S} & \cdots & \boldsymbol{\Lambda}\left(\Delta \tau_{D}\right) \mathbf{S}
\end{array}\right], \\
& \mathbf{W}_{P}=\left[\begin{array}{llll}
W_{P}(0) & W_{P}(1) & \cdots & W_{P}\left(K_{x_{P}}-1\right)
\end{array}\right]^{T} .
\end{aligned}
$$




\section{About Models and Colored Noise}

In the above two subsections, the time delay estimations in (2) and (8) are converted into sinusoidal parameter estimation problems as in (4) and (10), where $\left|R_{A}(\tau)\right|^{2}$ and $\left|R_{P}(\tau)\right|^{2}$ can be regarded as the power spectral densities of $x_{A}(k)$ and $x_{P}(k)$ as in (5) and (11), respectively. In later sections, we will propose a MUSIC-type algorithm to estimate $\tau_{i}$ in (4) and $\Delta \tau_{i}$ in (10). Following common practices as in [4]-[6], we assume the SNRs of the received signals in (2) and (8) are high, thus neglecting the colored noises $S(k) \cdot W^{*}(k)$ in (4) and $W_{P}$ in (10) (see [5],[12] and references therein) in algorithm derivation.

\section{Time Delay Estimation}

The time delay estimation problem in (4) and (10) is a parameter estimation of sinusoidal signals with lowpass envelopes [13]-[15]. The data models in (4) and (10) contain a multiplicative noise term $|S(k)|^{2}$. When the conventional MUSIC algorithm is applied to this case, Besson and Stoica [16] have shown that the estimator performance degrades slightly with very slowly varying envelopes, but the degradation is significant when the envelope fluctuation is not very slow. Thus the conventional methods should be modified to take into account the envelope variation.

\section{A. The Time Delay Estimation in Active Systems}

1) Multiple-experiment Data: Since $|S(k)|^{2}$ is the power spectral density of $s(t)$ and deterministic, the covariance matrix of $\mathbf{x}_{A}$ in (6) can be written as

$$
\mathbf{R}_{\mathbf{x}_{A}}=E\left(\mathbf{x}_{A} \mathbf{x}_{A}^{H}\right)=\boldsymbol{\Phi}_{A} E\left(\boldsymbol{\lambda}_{A} \boldsymbol{\lambda}_{A}^{H}\right) \boldsymbol{\Phi}_{A}^{H}+\mathbf{R}_{\mathbf{W}_{A}},
$$

where $\mathbf{R}_{\mathbf{W}_{A}}$ is colored noise matrix and defined as

$$
\begin{aligned}
\mathbf{R}_{\mathbf{W}_{A}} & =\left\{E\left[\left(\sum_{i=1}^{D} \lambda_{i}^{*} \boldsymbol{\Lambda}\left(\tau_{i}\right) \mathbf{S}\right) \mathbf{W}_{A}^{H}\right]\right. \\
+E & {\left.\left[\mathbf{W}_{A}\left(\sum_{i=1}^{D} \lambda_{i}^{*} \boldsymbol{\Lambda}\left(\tau_{i}\right) \mathbf{S}\right)^{H}\right]+E\left[\mathbf{W}_{A} \mathbf{W}_{A}^{H}\right]\right\} . }
\end{aligned}
$$

Since $|S(k)|^{2}$ is always non-zero and the time of arrivals $\tau_{1}, \tau_{2}, \cdots, \tau_{D}$ are different from each other, we have $\operatorname{rank}\{\boldsymbol{\Phi}\}=D$. Under the assumption that $\lambda_{1}, \lambda_{2}, \cdots, \lambda_{D}$ are mutually uncorrelated, $\operatorname{rank}\left\{E\left(\boldsymbol{\lambda}_{A} \boldsymbol{\lambda}_{A}^{H}\right)\right\}=D$. Then we have the following criterion [15] for estimating $\tau_{i}, i=$ $1,2, \cdots, D$, as in [17]

$$
\mathbf{\Phi}_{A}^{H} \mathbf{G}_{A} \cong \mathbf{0},
$$

where $\mathbf{G}_{A}$ is the noise subspace corresponding to $\mathbf{R}_{\mathbf{x}_{A}}$. The approximation in (14) arises from $\mathbf{R}_{\mathbf{W}_{A}}$.

2) Single-experiment Data: In many cases, multipleexperiment data may not be available. Thus, $\operatorname{rank}\left(\mathbf{x}_{A} \mathbf{x}_{A}^{H}\right)=1$ if $\mathbf{x}_{A}$ is obtained from single-experiment data, which makes the above method incapable of resolving multipath components. Here we will present a method to estimate $\tau_{i}, i=$ $1,2, \cdots, D$ in the case of single-experiment data.

The estimate of covariance matrix $\mathbf{R}_{\mathbf{x}_{A}}$ in the case of singleexperiment data can be expressed as [18]

$$
\hat{\mathbf{R}}_{\mathbf{x}_{A}}=\sum_{i=1}^{D}\left|\lambda_{i}\right|^{2} \hat{\mathbf{R}}_{\tilde{\mathbf{\Phi}}_{A i}}+\hat{\mathbf{R}}_{\mathbf{W}_{A}}=\hat{\mathbf{R}}_{\mathbf{S}_{A}}+\hat{\mathbf{R}}_{\mathbf{W}_{A}}
$$

where

$$
\begin{aligned}
& \hat{\mathbf{R}}_{\tilde{\Phi}_{A i}}=\tilde{\boldsymbol{\Phi}}_{A}\left(\tau_{i}\right) \tilde{\boldsymbol{\Phi}}_{A}^{H}\left(\tau_{i}\right) /\left(K_{x_{A}}-M_{A}+1\right) \\
& =\sum_{q=0}^{K_{x_{A}}-M_{A}}\left(\tilde{\phi}_{A}\left(q, \tau_{i}\right) \tilde{\phi}_{A}^{H}\left(q, \tau_{i}\right)\right) /\left(K_{x_{A}}-M_{A}+1\right) \text {, } \\
& \tilde{\boldsymbol{\Phi}}_{\sim_{\sim}}\left(\tau_{i}\right)= \\
& {\left[\begin{array}{llll}
\tilde{\phi}_{A}\left(0, \tau_{i}\right) & \tilde{\phi}_{A}\left(1, \tau_{i}\right) & \cdots & \tilde{\phi}_{A}\left(K_{x_{A}}-M_{A}, \tau_{i}\right)
\end{array}\right],} \\
& \tilde{\boldsymbol{\phi}}_{A}\left(q, \tau_{i}\right)=\left[|S(q)|^{2} e^{j 2 \pi \frac{q}{K_{x_{A}}} \tau_{i}}|S(q+1)|^{2} e^{j 2 \pi \frac{(q+1)}{K_{x_{A}}} \tau_{i}}\right. \\
& \left.\cdots\left|S\left(q+M_{\mathrm{A}}-1\right)\right|^{2} e^{j 2 \pi \frac{(q+M-1)}{K_{x_{A}}} \tau_{i}}\right]^{T} \\
& q=0,1, \cdots, K_{x}-M_{A},
\end{aligned}
$$

and $\hat{\mathbf{R}}_{\mathbf{W}_{A}}$ is the estimated covariance matrix of noise. We simply write $\tilde{\boldsymbol{\Phi}}_{A i}$ instead of $\tilde{\boldsymbol{\Phi}}_{A}\left(\tau_{i}\right)$ whenever there is no confusion. According to the common assumptions of the MUSIC algorithm in [18], it is necessary to have $K_{x_{A}}-M_{A}+1>M_{A}$ and $M_{A}>D$, or equivalently $\left(K_{x_{A}}+1\right) / 2>M_{A}>D$.

In general, the rank of $\hat{\mathbf{R}}_{\tilde{\mathbf{\Phi}}_{A i}}$ in (15) is larger than 1 and sometimes equals $M_{A}$ [15], [19]. Even though the covariance matrix for the $i$ th signal is full rank, the signal energy usually concentrates on the largest eigenvalue [15],[19]. The remaining dimensions of the matrix can be regarded as the quasi-noise subspace. Here, the concentration largely depends on $|S(k)|^{2}$ in (4) and (9) [13]-[15], [19]. Due to the orthogonality between signal and noise subspaces, there is

$$
\hat{\mathbf{R}}_{\mathbf{S}_{A}} \hat{\mathbf{G}}_{A} \cong 0
$$

where $\hat{\mathbf{G}}_{A}$ is the noise subspace corresponding to $\hat{\mathbf{R}}_{\mathbf{x}_{A}}$. Here the approximation also arises from the colored noise $\hat{\mathbf{R}}_{\mathbf{W}_{A}}$. In practice, we can determine the approximate quasi-signal subspace dimension by examining the number of dominant eigenvalues. In this paper we assume that the number of signal components is known and only address the parameter estimation. Since $\hat{\mathbf{R}}_{\mathbf{S}_{A}}$ is a linear combination of $\hat{\mathbf{R}}_{\tilde{\mathbf{\Phi}}_{A i}}$, we have

$$
\hat{\mathbf{R}}_{\tilde{\mathbf{\Phi}}_{A i}} \hat{\mathbf{G}}_{A} \cong 0
$$

By premultiplication of $\hat{\mathbf{G}}_{A}^{H}$ with (17), we have

$$
\hat{\mathbf{G}}_{A}^{H} \hat{\mathbf{R}}_{\tilde{\boldsymbol{\Phi}}_{A i}} \hat{\mathbf{G}}_{A}=\hat{\mathbf{G}}_{A}^{H} \tilde{\boldsymbol{\Phi}}_{A i} \tilde{\boldsymbol{\Phi}}_{A i}^{H} \hat{\mathbf{G}}_{A} \cong 0 .
$$

Therefore, we have the following relation

$$
\tilde{\boldsymbol{\Phi}}_{A i}^{H} \hat{\mathbf{G}}_{A} \cong \mathbf{0}, \quad i=1,2, \cdots, D
$$

Then we denote

$$
P_{A}(\tau)=\frac{1}{\tilde{\mathbf{S}}^{H} \tilde{\Lambda}_{A}^{H}(\tau) \hat{\mathbf{G}}_{A}},
$$

where $\tilde{\boldsymbol{\Lambda}}_{A}(\tau)=\operatorname{diag}\left(\begin{array}{cccc}1 & e^{\frac{2 \pi \tau}{K_{x}}} & \ldots & e^{\frac{2 \pi\left(M_{A}-1\right) \tau}{K_{x}}}\end{array}\right), \tilde{\mathbf{S}}$ is an $M_{A} \times 1$ sub-vector formed by $|S(k)|^{2}$. In our simulations, we always take the segment that contains the main spectral components of $s(n)$. Since $|S(k)|^{2}$ is known, we can perform a 1-dimensional search with respect to $\tau$ to find the maxima of (20), whose locations are the time delay estimates $\hat{\tau}_{i}, i=$ $1,2, \cdots, D$. 
In this paper, since we have the power spectrum of $\mathbf{x}_{A}$ as in (5), we adopt the idea in [21] to estimate the covariance matrix from single-experiment data, instead of that in (15). We first obtain the correlation function $R_{x_{A}}^{\prime}(l)$ from $\left|\bar{R}_{A}(\tau)\right|^{2}$ according to the Wiener-Khinchine theorem [20]

$$
R_{x_{A}}^{\prime}(l)=\mathrm{DFT}^{-1}\left[\left|\bar{R}_{A}(\tau)\right|^{2}\right], \quad l=0,1, \cdots, 2 K_{x_{A}}-1 .
$$

where $\left|\bar{R}_{A}(\tau)\right|^{2}$ is a sequence formed by zero-padding $\left|R_{A}(\tau)\right|^{2}$ to length $2 K_{x_{A}}-1$. Thus, $R_{x_{A}}^{\prime}(l)$ can be viewed as the correlation function of an analytic signal. Then the covariance matrix can be formed based on $R_{x_{A}}^{\prime}(l)$ [21]. The dimension of the covariance matrix is decreased to $M_{A}$ to get the following truncated covariance matrix

$$
\hat{\mathbf{R}}_{\mathbf{x}_{A}}^{\prime}=\left[R_{A i j}^{\prime}\right]_{M_{\mathbf{A}} \times M_{\mathbf{A}}},
$$

where $R_{A i j}^{\prime}=R_{x_{A}}^{\prime}(i-j)$ for $i \geq j$, and $R_{A j i}^{\prime}=R_{A i j}^{\prime *}$ for $i<j$. It has been shown that $\hat{\mathbf{R}}_{\mathbf{x}_{A}}$ in (15) and $\hat{\mathbf{R}}_{x_{A}}^{\prime}$ in (22) are the consistent estimates of $\mathbf{R}_{\mathbf{x}_{A}}$ [20]. Thus, we can substitute $\hat{\mathbf{R}}_{x_{A}}^{\prime}$ for $\hat{\mathbf{R}}_{\mathbf{x}_{A}}$ in our algorithm.

\section{B. The Time Delay Estimation in Passive Systems}

In passive systems, if the transmitted signal is known, the time delay $\Delta \tau_{i}, i=1,2, \cdots, D$, in (12) can also be estimated by the above method. However, the transmitted signal in passive systems is always unknown. Therefore, the minimization requires the search not only on $\Delta \tau$, but also on the unknown transmitted signal. Thus the above method can not be directly applied to obtain $\Delta \hat{\tau}_{i}, i=1,2, \cdots, D$. However, in the case of single-experiment data, we still have

$$
\tilde{\boldsymbol{\Phi}}_{P i}^{H} \hat{\mathbf{G}}_{P} \cong 0
$$

where

$$
\begin{aligned}
& \tilde{\boldsymbol{\Phi}}_{P i} \triangleq \tilde{\boldsymbol{\Phi}}_{P}\left(\Delta \tau_{i}\right)= \\
& {\left[\begin{array}{llll}
\tilde{\phi}_{P}\left(0, \Delta \tau_{i}\right) & \tilde{\phi}_{P}\left(1, \Delta \tau_{i}\right) & \cdots & \tilde{\phi}_{P}\left(K_{x_{P}}-M_{P}, \Delta \tau_{i}\right)
\end{array}\right],} \\
& \tilde{\boldsymbol{\phi}}_{P}\left(q, \Delta \tau_{i}\right)=\left[|S(q)|^{2} e^{j 2 \pi \frac{q}{K_{x_{P}}} \Delta \tau_{i}}|S(q+1)|^{2} e^{j 2 \pi \frac{(q+1)}{K_{x_{P}}} \Delta \tau_{i}}\right. \\
& \left.\cdots\left|S\left(q+M_{\mathrm{P}}-1\right)\right|^{2} e^{j 2 \pi \frac{\left(q+M_{P}-1\right)}{K_{x_{P}}} \Delta \tau_{i}}\right]^{T}, \\
& q=0,1, \cdots, K_{x_{P}}-M_{P},
\end{aligned}
$$

and $\hat{\mathbf{G}}_{P}$ is the noise subspace corresponding to the estimated covariance matrix $\hat{\mathbf{R}}_{x_{P}}$ of $\mathbf{x}_{P}$. Similar to that in active systems, $\hat{\mathbf{R}}_{x_{P}}$ is substituted by $\hat{\mathbf{R}}_{x_{P}}^{\prime}$, which can be obtained as in (24) and (25):

$$
R_{x_{P}}^{\prime}(l)=\mathrm{DFT}^{-1}\left[\left|\bar{R}_{P}(\tau)\right|^{2}\right], \quad l=0,1, \cdots, 2 K_{x_{P}}-1,
$$

where $\left|\bar{R}_{P}(\tau)\right|^{2}$ is a sequence produced by zero-padding $\left|R_{P}(\tau)\right|^{2}$ to length $2 K_{x_{P}}-1$.

$$
\hat{\mathbf{R}}_{\mathbf{x}_{P}}^{\prime}=\left[R_{P i j}^{\prime}\right]_{M_{P} \times M_{P}},
$$

where $R_{P i j}^{\prime}=R_{x_{P}}^{\prime}(i-j)$ for $i \geq j$, and $R_{P j i}^{\prime}=R_{P i j}^{*}$ for $i<j$. It is necessary to have $\left(K_{x_{P}}+1\right) / 2>M_{P}>2 D$ [15].

Here the time delay estimation can be further expressed as $\left(\Delta \hat{\tau}_{i}, \tilde{\mathbf{S}}\right)=\arg \min _{(\Delta \tau, \boldsymbol{\Psi})}\left\{\boldsymbol{\Psi}^{H} \tilde{\boldsymbol{\Lambda}}_{P}^{H}(\Delta \tau) \hat{\mathbf{G}}_{P} \hat{\mathbf{G}}_{P}^{H} \tilde{\boldsymbol{\Lambda}}_{P}(\Delta \tau) \boldsymbol{\Psi}\right\}$

where $\Psi$ is a vector of real-valued variables representing the power spectrum of the unknown transmitted signal $s(t)$,

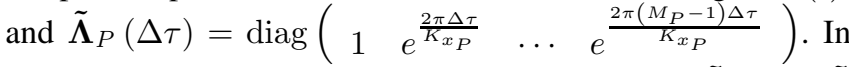
theory, a perfect estimate on $\Psi$ should be equal to $\tilde{\mathbf{S}}$. Since $\tilde{\mathbf{S}}$ and $\left\{\boldsymbol{\Psi}^{H} \tilde{\boldsymbol{\Lambda}}_{P}^{H}(\Delta \tau) \hat{\mathbf{G}}_{P} \hat{\mathbf{G}}_{P}^{H} \tilde{\boldsymbol{\Lambda}}_{P}(\Delta \tau) \boldsymbol{\Psi}\right\}$ are real-valued, but $\tilde{\boldsymbol{\Lambda}}_{P}^{H}(\Delta \tau) \hat{\mathbf{G}}_{P} \hat{\mathbf{G}}_{P}^{H} \tilde{\boldsymbol{\Lambda}}_{P}(\Delta \tau)$ is complex-valued, there must be $\boldsymbol{\Psi}^{H} \operatorname{Im}\left\{\tilde{\boldsymbol{\Lambda}}_{P}^{H}(\Delta \tau) \hat{\mathbf{G}}_{P} \hat{\mathbf{G}}_{P}^{H} \tilde{\boldsymbol{\Lambda}}_{P}(\Delta \tau)\right\} \boldsymbol{\Psi}=0$, and we have

$$
\begin{aligned}
& \Psi^{H} \tilde{\boldsymbol{\Lambda}}_{P}^{H}(\Delta \tau) \hat{\mathbf{G}}_{P} \hat{\mathbf{G}}_{P}^{H} \tilde{\boldsymbol{\Lambda}}_{P}(\Delta \tau) \boldsymbol{\Psi} \\
= & \Psi^{H} \operatorname{Re}\left\{\tilde{\boldsymbol{\Lambda}}_{P}^{H}(\Delta \tau) \hat{\mathbf{G}}_{P} \hat{\mathbf{G}}_{P}^{H} \tilde{\boldsymbol{\Lambda}}_{P}(\Delta \tau)\right\} \boldsymbol{\Psi} .
\end{aligned}
$$

The operation of taking the real part in (27) is necessary [15]. Thus (26) is further written as

$$
\left(\Delta \hat{\tau}_{i}, \tilde{\mathbf{S}}\right)=\arg \min _{(\Delta \tau, \Psi)}\left\{\boldsymbol{\Psi}^{H} \mathbf{\Theta}(\Delta \tau) \boldsymbol{\Psi}\right\}
$$

where $\boldsymbol{\Theta}(\Delta \tau)=\operatorname{Re}\left\{\tilde{\boldsymbol{\Lambda}}_{P}^{H}(\Delta \tau) \hat{\mathbf{G}}_{P} \hat{\mathbf{G}}_{P}^{H} \tilde{\boldsymbol{\Lambda}}_{P}(\Delta \tau)\right\}$. The solution to (28) is a general optimization problem [22]. To estimate the time delay by using optimization, some constraints are imposed. Since $\tilde{\mathbf{S}}>\mathbf{0}$, it is reasonable to require $\boldsymbol{\Psi}>\mathbf{0}$ [15] to avoid pseudo estimates [14], where $\mathbf{0}=\left[\begin{array}{llll}0 & 0 & \cdots & 0\end{array}\right]^{T}$ is an $M_{P} \times 1$ column vector. Then the time delay estimates can be expressed as

$$
\begin{aligned}
\left(\Delta \hat{\tau}_{i}, \tilde{\mathbf{S}}\right)=\arg \min _{(\Delta \tau, \Psi)}\left\{\boldsymbol{\Psi}^{H} \boldsymbol{\Theta}(\Delta \tau) \boldsymbol{\Psi}\right\}, \\
\text { s.t. } \quad \boldsymbol{\Psi}^{H} \boldsymbol{\Psi}=1,
\end{aligned}
$$

where $\Psi^{H} \Psi=1$ is a nonlinear constraint and normalization. Since $\left\{\boldsymbol{\Psi}^{H} \boldsymbol{\Theta}(\Delta \tau) \boldsymbol{\Psi}\right\}$ is nonnegative for any real $\boldsymbol{\Psi}, \Theta(\Delta \tau)$ is a nonnegative definite Hermitian matrix, which ensures the global convergence of the quadratic form $\left\{\boldsymbol{\Psi}^{H} \boldsymbol{\Theta}(\Delta \tau) \boldsymbol{\Psi}\right\}$ [22].

The parameter estimation in (29) can be done in two steps. First, we minimize $\left\{\boldsymbol{\Psi}^{H} \boldsymbol{\Theta}(\Delta \tau) \boldsymbol{\Psi}\right\}$ with respect to $\boldsymbol{\Psi}$, and yield $\xi_{\min }(\Delta \tau)$ as a function of $\Delta \tau$

$$
\begin{aligned}
\xi_{\min }(\Delta \tau)= & \min _{(\boldsymbol{\Psi})}\left\{\boldsymbol{\Psi}^{H} \boldsymbol{\Theta}(\Delta \tau) \boldsymbol{\Psi}\right\}, \\
\text { s.t. } & \boldsymbol{\Psi}^{H} \boldsymbol{\Psi}=1, \\
& \boldsymbol{\Psi}>\mathbf{0} .
\end{aligned}
$$

Then, a search step is performed on $\Delta \tau$ to find the minima of $\xi_{\min }(\Delta \tau)$

$$
\Delta \hat{\tau}_{i}=\arg \min _{(\Delta \tau)}\left\{\xi_{\min }(\Delta \tau)\right\},
$$

where $\Delta \hat{\tau}_{i}, i=1,2, \cdots, D$, are the estimates of $\Delta \tau_{i}$.

\section{Performance and Simulation Results}

In this section, we evaluate the performance [1], [23] of the proposed methods. 


\section{A. Transmitted and Received Signals}

In our simulations, the transmitted signal is a chirp signal

$$
\begin{aligned}
& s(n)=\sin \left((\beta \cdot n+\alpha) \cdot n+\varphi_{0}\right), \\
& n=0,1, \cdots, K_{s}-1,
\end{aligned}
$$

where $\beta=\pi \cdot\left(f_{2}-f_{1}\right) / K_{s}, f_{2}$ and $f_{1}$ are the upper and lower frequencies of $s(n)$, respectively, $\alpha=2 \pi \cdot f_{1}, \varphi_{0}$ is the initial random phase, $K_{s}$ is the length of $s(n)$. The bandwidth of $s(n)$ is defined as $B_{s} \triangleq\left(f_{2}-f_{1}\right)$, and the correlation time is $\zeta \triangleq 1 / B_{s}$. In our simulations, $s(n)$ can not be considered as having flat spectrum since $B_{s} \ll 0.5$.

In active systems, we assume the received signal in (2) has two signal components with the same valued amplitudes. Then the received signals are modeled as

$$
\begin{aligned}
r(n)=\lambda \cdot s\left(n-\tau_{1}\right)+\lambda \cdot s & \left(n-\tau_{2}\right)+w(n), \\
& n=0,1, \cdots, K_{r}-1,
\end{aligned}
$$

where $\tau_{1}$ and $\tau_{2}$ are the unknown time delays. In the received signal $r(n)$, both $s\left(n-\tau_{1}\right)$ and $s\left(n-\tau_{2}\right)$ are padded with zeros to length $K_{r}$ and nonzero values only occupy the range $\tau_{1} \leq n \leq \tau_{1}+K_{s}-1$ and $\tau_{2} \leq n \leq \tau_{2}+K_{s}-1$, respectively. Let $\Delta D_{A}=\left|\tau_{2}-\tau_{1}\right|$ denote the time delay separation.

In passive systems, we assume the received signals can be modeled as

$$
\left\{\begin{array}{c}
r_{1}(n)=\lambda \cdot s(n)+w_{1}(n) \\
r_{2}(n)=\lambda \cdot s\left(n-\tau_{21}\right)+\lambda \cdot s\left(n-\tau_{22}\right)+w_{2}(n) \\
n=0, \cdots, K_{r}-1,
\end{array}\right.
$$

where $\tau_{11}=0, \tau_{21}$ and $\tau_{22}$ are the unknown time delays. Then $\Delta \tau_{1}=\tau_{21}$ and $\Delta \tau_{2}=\tau_{22}$ are the time differences of arrival to be estimated. In the received signal $r_{1}(n), s(n)$ is padded with zeros to length $K_{r}$ and nonzero values only occupy the range $0 \leq n \leq K_{s}-1$. In the received signal $r_{2}(n)$, both $s\left(n-\tau_{21}\right)$ and $s\left(n-\tau_{22}\right)$ are padded with zeros to length $K_{r}$ and nonzero values only occupy the range $\tau_{21} \leq n \leq$ $\tau_{21}+K_{s}-1$ and $\tau_{22} \leq n \leq \tau_{22}+K_{s}-1$, respectively. Let $\Delta D_{P}=\left|\Delta \tau_{2}-\Delta \tau_{1}\right|$ denote the separation of the time differences of arrival.

\section{B. Simulation Results}

Fig. 1 and Fig. 2 show the MSE's of the time delay estimation for different SNRs and time delay separations $\left(\Delta D_{A}\right.$ and $\left.\Delta D_{P}\right)$, which are also compared with CRB. The results are obtained through 500 independent Monte Carlo trials. SNR is defined as $10 \log _{10}\left(P_{s} / P_{n}\right)$, where $P_{s}$ is the power of the signal, $P_{n}$ is the power of the noise. Since the CRBs of the time delay estimates degrade slightly due to the closely spaced sources as in (33) and (34) for the chirp signal, we do not consider the difference in our simulations. We note that the MSEs are close to the corresponding CRBs over a wide range of SNRs. Further, there is slight performance variation for different $\Delta D_{A}$ and $\Delta D_{P}$.

In Fig. 3 and Fig. 4, we compare the performance of a MUSIC-type algorithm with the conventional MUSIC algorithm [2], [6] by using only a signal component. It is clear that our MUSIC-type algorithm outperforms the convention MUSIC algorithm for all bandwidth values. The performance gap is especially noticeable for small bandwidth values (corresponding to high variations of the power spectrum envelope $|S(k)|^{2}$ in (4) and (10) ), where the conventional MUSIC algorithm has severe performance degradations. The better performance of MUSIC-type algorithm comes from the matching of the data models in (4) and (10). The results in Fig. 3 and Fig. 4 are consistent with the conclusions in [16].

In Fig. 5 and Fig. 6, we compare the MUSIC-type algorithm with the correlation approach and the conventional MUSIC algorithm. We set $f_{1}=0.3, f_{2}=0.33$, and $\Delta D_{A}=\Delta D_{P}=3$. Then $\Delta D_{A}=\Delta D_{P} \ll \zeta \cong 33$. We have two observations. First, the correlation approach is unable to resolve the two closely-spaced signal components. Second, MUSIC-type algorithm has a satisfactory estimation quality and is better than the conventional MUSIC algorithm.

\section{CONCLUSIONS}

In this paper, we have studied the super-resolution time delay estimation. Two cases, active and passive systems, are considered. After transforming the time delay estimation into a sinusoidal parameter estimation problem, we propose a superresolution time delay estimation method by generalizing the MUSIC algorithm from the single-experiment data. The time delay estimates are improved since the multiplicative noise is taken into account. Simulation results show that the MSE of the time delay estimates for different SNRs are very close to the CRB over a wide range of SNRs. In comparison with the conventional MUSIC algorithm and the correlation approach, the MUSIC-type algorithm has better performance.

\section{REFERENCES}

[1] A. H. Quazi, "An overview on the time delay estimate in active and passive systems for target localization," IEEE Trans. ASSP, vol. 29, no. 3, pp. 527-533, Jun. 1981 .

[2] M. C. Vanderveen, A.-J. Van der Veen, and A. Paulraj, "Estimation of multipath parameters in wireless communications," IEEE Trans. SP, vol. 46, no. 3, pp. 682-690, 1998.

[3] A. M. Bruckstein, T. J. Shan, and T. Kailath, "The resolution of overlapping echoes," IEEE Trans. ASSP, vol. 33, no. 6, pp. 1357-1367, 1985.

[4] Z. Q. Hou and Z. D. Wu, "A new method for high resolution estimation of time delay," Proc. ICASSP, 1982, pp. 420-423.

[5] M. Pallas and G. Jourdain, "Active high resolution time delay estimation for large BT signals," IEEE Trans. SP, vol. 39, no. 4, pp.781-787, 1991

[6] M. A. Hasan, M. R. Azimi-Sadjadi, and G.J. Dobeck, "Separation of multiple time delays using new spectral estimation schemes," IEEE Trans. SP, vol. 46, no. 6, pp.1580-1590, 1998.

[7] T. Manabe and H. Takai, "Superresolution of multipath delay profiles measured by PN correlation method," IEEE Trans. AP, vol. 40, no. 5, pp. 500-509, May. 1992.

[8] J. P. Ianniello, "High-resolution multipath time delay estimation for broad-band random signals," IEEE Trans. ASSP, vol. 36, no. 3 , pp. $320-327,1988$.

[9] M. Feder and E. Weinstein, "Parameter estimation of superimposed signals using the EM algorithm," IEEE Trans. ASSP, vol. 36, no. 2, pp. 477-489, 1988.

[10] R. B. Wu and J. Lian, "Time-delay estimation via optimization highly oscillatory cost functions," IEEE Journal of Oceanic Engineering, vol. 23, no. 3, pp. 235-244, 1998.

[11] P. P. Moghaddam, H. Amindavar, and R. L. Kirlin, "A new time-delay estimation in multipath," IEEE Trans. SP, vol. 51, no. 5, pp. 1129-1142, 2003. 
[12] W. Chen, G. Zhou, and G. B. Giannakis, "Velocity and acceleration estimation of Doppler weather radar/lidar signals in colored noise," Proc. ICASSP, 1995, pp. 2052-2055.

[13] F. X. Ge, Q. Wan, X. T. Wang, and Y. N. Peng, "Frequency estimation of the sinusoidal signals with lowpass envelopes based on the eigenanalysis," Proc. IEEE Radar Conference, CA., USA, Apr. 2002, pp. 453-458.

[14] F. X. Ge, H. Zhang, J. Yang, and Y. N. Peng, "Super-resolution frequency estimation of the sinusoidal signals with unknown lowpass envelopes,' Proc. IEEE Radar Conference, AL., USA, May 2003, pp. 273- 277.

[15] F. X. Ge, The Parameter Estimation for Wireless Location in Cellular Communication System, Ph.D dissertation, Tsinghua University, 2003.

[16] O. Besson, and P. Stoica, "Analysis of MUSIC and ESPRIT frequency estimates for sinusoidal signals with lowpass envelopes," IEEE Trans. SP, vol. 44, no. 9, pp. 2359-2364, 1996.

[17] R. O. Schmidt, "Multiple emitter location and signal parameter estimation," IEEE Trans. AP, vol. 34, no. 3, pp. 276-280, 1986.

[18] P. Stoica and A. Nehorai, "MUSIC, maximum likelihood, and CramerRao bound," IEEE Trans. ASSP, vol. 37, no. 5, pp. 720-741, 1989.

[19] Y. Meng, P. Stoica, and K. M. Wong, "Estimation of the Directions of Arrival of Spatially Dispersed Signals in Array Processing," IEE Proceedings-Radar, Sonar and Navigation, vol. 143, no. 1 pp. 1-9, 1996.

[20] J. G. Proakis and D. G. Manolakis, Digital signal processing : principles, algorithms, and applications, 3rd ed., N.J. : Prentice Hall, 1996.

[21] Q. S. Ren and A. J. Willis, "Extending MUSIC to single snapshot and on line direction finding applications," IEE Conference Publication, Radar 97, pp. 783-787.

[22] M. A. Bhatti, Practical optimization methods: with Mathematica applications, New York: Springer, 2000.

[23] A. Zeira and P. M. Schultheiss, "Time delay estimation for closely spaced echoes," Proc. ICASSP, 1990, pp. 2763-2766.

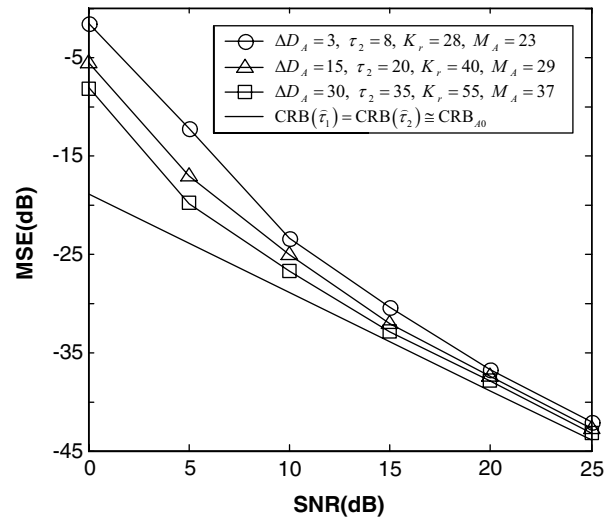

Fig. 1. $\mathrm{CRB}$ and MSE of time delay estimation versus SNR and $\Delta D_{A}$ in active systems $\left(K_{s}=20, \tau_{1}=5, f_{1}=0.30, f_{2}=0.33\right)$

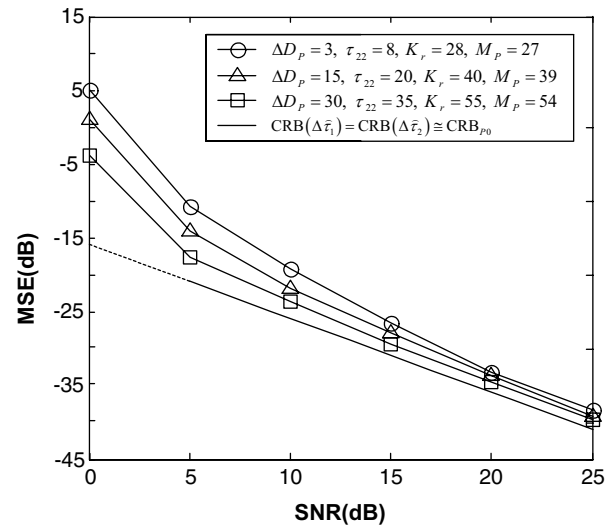

Fig. 2. $\mathrm{CRB}$ and MSE of time delay estimation versus SNR and $\Delta D_{P}$ in passive systems $\left(K_{s}=20, \tau_{21}=5, f_{1}=0.30, f_{2}=0.33\right)$

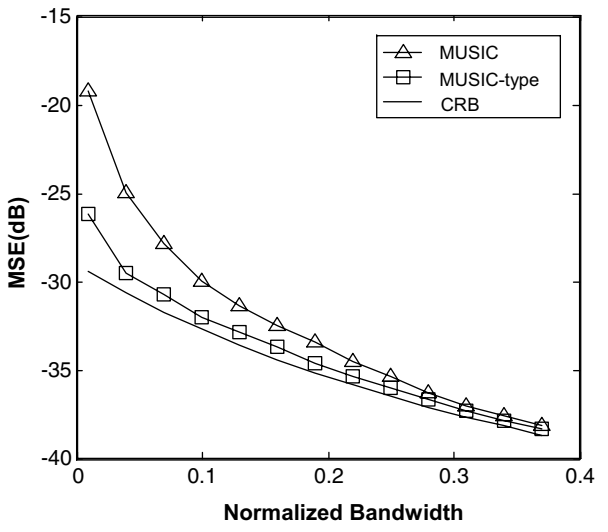

Fig. 3. CRB and MSE of time delay estimation versus normalized bandwidth in active systems $\left(K_{s}=20, K_{r}=28, \mathrm{SNR}=20 \mathrm{~dB}, M_{A}=23, f_{1}=0.1\right)$

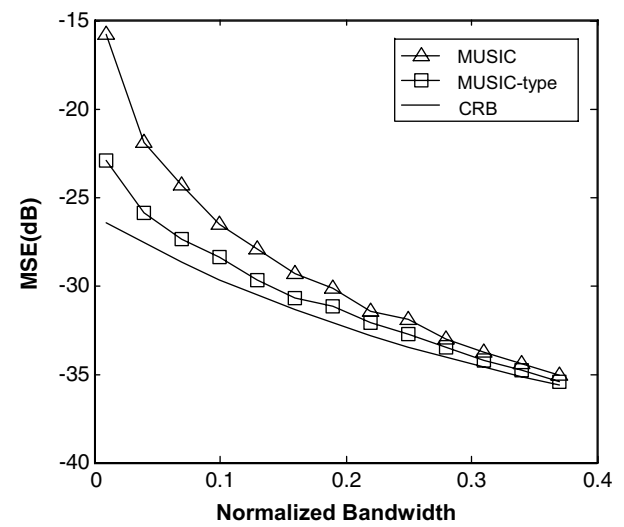

Fig. 4. CRB and MSE of time delay estimation versus normalized bandwidth in passive systems $\left(K_{s}=20, K_{r}=28, \mathrm{SNR}=20 \mathrm{~dB}, M_{P}=27, f_{1}=0.1\right)$

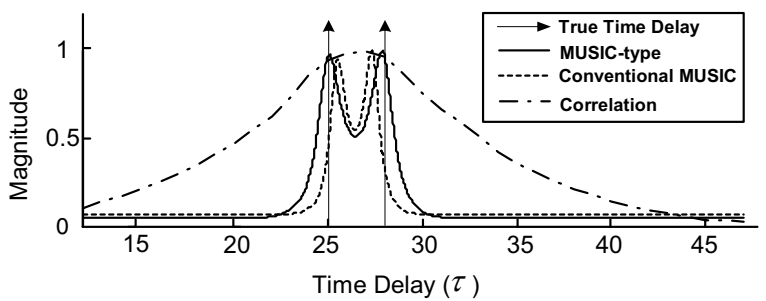

Fig. 5. Performance comparison of time delay estimation in active systems $\left(K_{s}=20, K_{r}=28, \mathrm{SNR}=15 \mathrm{~dB}, M_{A}=23, \tau_{1}=5, \tau_{2}=8\right)$

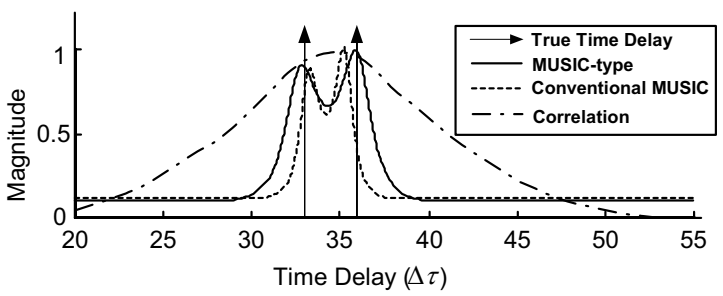

Fig. 6. Performance comparison of time delay estimation in passive systems $\left(K_{s}=20, K_{r}=28, \mathrm{SNR}=15 \mathrm{~dB}, M_{P}=27, \tau_{21}=5, \tau_{22}=8\right)$ 